\title{
Intelligent Modification of Colors in Digitized Paintings for Enhancing the Visual Perception of Color-blind Viewers
}

Paul Doliotis, George Tsekouras, Christos-Nikolaos Anagnostopoulos, and Vassilis Athitsos

\begin{abstract}
Color vision deficiency (CVD) is quite common since 8\%-12\% of the male and $0.5 \%$ of the female European population seem to be color-blind to some extent. Therefore there is great research interest regarding the development of methods that modify digital color images in order to enhance the color perception by the impaired viewers. These methods are known as daltonization techniques. This paper describes a novel daltonization method that targets a specific type of color vision deficiency, namely protanopia. First we divide the whole set of pixels into a smaller group of clusters. Subsequently we split the clusters into two main categories: colors that protanopes (persons with protanopia) perceive in a similar way as the general population, and colors that protanopes perceive differently. The color clusters of the latter category are adapted in order to improve perception, while ensuring that the adapted colors do not conflict with colors in the first category. Our experiments include results of the implementation of the proposed method on digitized paintings, demonstrating the effectiveness of our algorithm.
\end{abstract}

\section{Introduction}

Color vision deficiency (CVD) is quite common since $8 \%-12 \%$ of the male and $0.5 \%$ of the female European population seem to be color-blind to some extent. There is still no known medical treatment for this kind of problem. People suffering from any type of color vision deficiency are not considered to be seriously disabled. However there are certain cases in the daily activities of those people where the different perception of colors could lead to experiences that range from simply an-

Paul Doliotis and Vassilis Athitsos

Computer Science and Engineering Department, University of Texas at Arlington, USA

George Tsekouras and Christos-Nikolaos Anagnostopoulos

Department of Culture, Technology, and Communication, University of the Aegean, Greece

Please use the following format when citing this chapter:

Doliotis, P., Tsekouras, G., Anagnostopoulos, C.-N. and Athitsos, V., 2009, in IFIP International Federation for Information Processing, Volume 296; Artificial Intelligence Applications and Innovations III; Eds. Iliadis, L., Vlahavas, I., Bramer, M.; (Boston: Springer), pp. 293-301. 
noying (e.g., when viewing artwork or browsing websites) to really dangerous (e.g., traffic signalling).

Several techniques have been proposed that modify digital color images in order to enhance the color perception and reduce the confusion by viewers with CVD. These methods are known as daltonization techniques. In this paper we describe a daltonization technique that targets a specific type of color vision deficiency, namely protanopia. In our method, we first divide the set of image colors into a smaller group of clusters, reducing the amount of distinct colors. Subsequently we split the clusters into two main categories: a category of colors that protanopes can perceive in the same way as the general population, and a category of colors that protanopes perceive differently. The color clusters belonging to the latter category are adapted according to some initial daltonization parameters. Additionally we employ a color checking module to make sure that there will not be any color confusion between the two aforementioned categories. If there is color confusion the daltonization parameters are being iteratively modified until the confusion diminishes.

Many researchers have conducted research for appropriately modeling the visual perception of persons with CVD. In [4], a daltonization technique is presented based on the work published in [11] in order to modify a digital image so that it is more visible to people with CVD. The former work is also compared to the online results that are obtained visiting the Vischeck site [3].

The problem of color adaptation according to the user's perception is also addressed in [9, 12]. In [9], one of the issues addressed was the problem of tailoring visual content within the MPEG-21 Digital Item Adaption (DIA) framework to meet the user's visual perception characteristics. The image retrieval aspect for people with CVD was discussed in [7]. A physiologically motivated human color visual system model which represents visual information with one brightness component and two chromatic components was proposed for testing the color perception of people suffering from CVD [8]. In [1] a method was proposed for automaticly adapting the daltonization parameters for each image, to ensure that there is no loss of image structure due to conflict among colors that were daltonized and colors that remained intact. The method described in this paper builds on top of [1], introducing a color clustering step in order to drastically improve the efficiency of color conflict detection.

\section{Image Daltonization}

The method is based on the LMS system, which specifies colors in terms of the relative excitations of the longwave sensitive (L), the middlewave sensitive (M), and the shortwave sensitive (S) cones. As dichromats lack one class of cone photopigment, they confuse colors that differ only in the excitation of the missing class of photopigment. In contrast to the case of the trichromatic observer, who perceives three color components, two components are sufficient to specify color for the dichromat. The color perception of dichromats can be modeled using simple color transformations. 
As in [3], the transformation from RGB to LMS color is obtained using a matrix $T_{1}$, defined as follows:

$$
T_{1}=\left[\begin{array}{ccc}
17.8824 & 43.5161 & 4.1193 \\
3.4557 & 27.1554 & 3.8671 \\
0.02996 & 0.18431 & 1.4670
\end{array}\right]
$$

Given matrix $T_{1}$, the transformation from RGB to LMS is defined as:

$$
\left[\begin{array}{lll}
L & M & S
\end{array}\right]^{t}=T_{1}\left[\begin{array}{lll}
R & G & B
\end{array}\right]^{t},
$$

where $X^{t}$ denotes the transpose of matrix $X$.

Protanopes perceive colors in a different way due to the lack of one class of cone photopigment. The different color perception of protanopes can be modeled as a linear transformation, mapping normal cone responses LMS to protanope cone responses $L_{p} M_{p} S_{p}$. This linear mapping is represented by a matrix $T_{2}$ defined as:

$$
T_{2}=\left[\begin{array}{ccc}
0 & 2.02344 & -2.52581 \\
0 & 1 & 0 \\
0 & 0 & 1
\end{array}\right]
$$

Using $T_{2}, L_{p} M_{p} S_{p}$ is computed as:

$$
\left[\begin{array}{lll}
L_{p} & M_{p} & S_{p}
\end{array}\right]^{t}=T_{2}\left[\begin{array}{lll}
L & M & S
\end{array}\right]^{t},
$$

Finally, the RGB color perception of protanopes is modeled using a matrix $T_{3}=$ $T_{1}^{-1}$ defined as:

$$
T_{3}=\left[\begin{array}{ccc}
0.0809 & -0.1305 & 0.1167 \\
-0.0102 & 0.0540 & -0.1136 \\
-0.0003 & -0.0041 & 0.6935
\end{array}\right]
$$

Given $T_{3}$, and given a color described by $R, G, B$, a protanope perceives that color as $R_{p}, G_{p}, B_{p}$, defined as follows:

$$
\left[\begin{array}{lll}
R_{p} & G_{p} & B_{p}
\end{array}\right]^{t}=T_{3}\left[\begin{array}{lll}
L_{p} & M_{p} & S_{p}
\end{array}\right]^{t}=T_{3} T_{2} T_{1}\left[\begin{array}{lll}
R & G & B
\end{array}\right]^{t}=T_{1}^{-1} T_{2} T_{1}\left[\begin{array}{lll}
R & G & B
\end{array}\right]^{t},
$$

A simulation of the colors perceived by a protanope is highlighted in Figures 1a and $1 \mathrm{~b}$. It is evident that reddish tones are confused with black and moreover if those shades are neighbouring they are perceived as one color, thus changing the perception of image structure.

Based on the above definitions, we can define error quantities $E_{R}, E_{G}, E_{B}$ that express the difference between the normal and the protanope perception of a color:

$$
\begin{aligned}
& E_{R}=\left|R-R_{p}\right| \\
& E_{G}=\left|G-G_{p}\right| \\
& E_{B}=\left|B-B_{p}\right|
\end{aligned}
$$




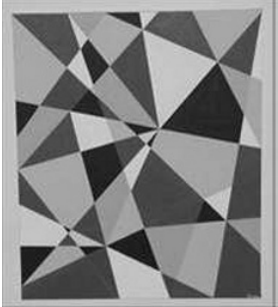

(a)

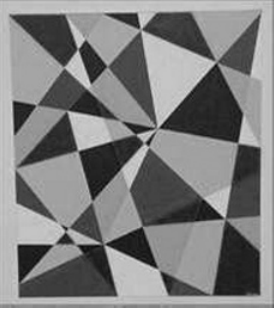

(b)

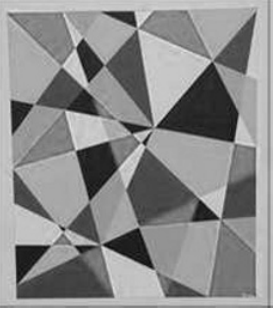

(c)

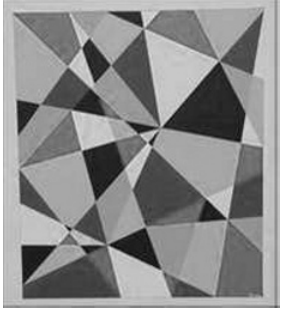

(d)

Fig. 1 (a) Original artwork ("Prism", modern abstract painting in acrylics by Bruce Gray [6]), (b) Simulation of the artwork as perceived by a protanope. Note the confusion of red and black areas in the artwork. www.brucegray.com/images/prism.jpg, (c) original image daltonized with $E_{\text {mod }}$, (d) protanope's perception of (c)

Following [4], these errors are added back to the original image, but in such a way that the error values are redistributed to the blue side of the spectrum, so as to improve the perception of that color by a protanope. In particular, if $E_{R}(i, j), E_{G}(i, j), E_{B}(i, j)$ are the error values at pixel $(i, j)$, these error values are converted to values $E_{R, \bmod }(i, j), E_{G, \bmod }(i, j), E_{B, \bmod }(i, j)$, which represent a shift of color $\left(E_{R}(i, j), E_{G}(i, j), E_{B}(i, j)\right)$ towards the blue side of the spectrum. This conversion is done using a matrix $M$ defined as follows:

$$
M=\left[\begin{array}{ccc}
0 & 0 & 0 \\
0.7 & 1 & 0 \\
0.7 & 0 & 1
\end{array}\right]
$$

Using $M, E_{R, \bmod }(i, j), E_{G, \bmod }(i, j), E_{B, \bmod }(i, j)$ are obtained as:

$$
\left[E_{R, \bmod }(i, j), E_{G, \bmod }(i, j), E_{B, \bmod }(i, j)\right]^{t}=M\left[E_{R}(i, j), E_{G}(i, j), E_{B}(i, j)\right]^{t} .
$$

Then, the daltonized color is obtained by adding the modified error back to the original color. At position $(i, j)$, given the color $R(i, j), G(i, j), B(i, j)$ of the original image, the color $R_{d}(i, j), G_{d}(i, j), B_{d}(i, j)$ in the daltonized image is defined as follows:

$$
\begin{aligned}
{\left[R_{d}(i, j), G_{d}(i, j), B_{d}(i, j)\right]=} & {[R(i, j), G(i, j), B(i, j)]+} \\
& {\left[E_{R, \bmod }(i, j), E_{G, \bmod }(i, j), E_{B, \bmod }(i, j)\right] . }
\end{aligned}
$$

Using the above method, good results may be achieved in the majority of color images as shown in Figures 1c and 1d. However, there is still a lot of space for improvements. The most obvious one is the fact that the adaptation parameters of Equation 10 are manually chosen. In addition, there is always the possibility that, in the daltonized image, some of the modified colors may still be confused with other colors in the image by a protanope, as for example in Figure 2. As a result, in addition to loss of color information there can be also loss of information about the 
image structure. For these important reasons, an intelligent daltonization method is proposed in [1], that uses an automatic iterative technique for the selection of the adaptation parameters. That method also takes into consideration a color checking module, so as to ensure that the modified colors, as perceived by a protanope, do not get confused with other intact colors in the image.

The method described in this paper builds on top of the method proposed in [1]. The key difference between our method and [1] is that, in this paper, a color clustering step is introduced, that drastically improves the efficiency of the color checking module. In the next section we describe the proposed daltonization method.

\section{Color Clustering and Selection of Daltonization Parameters}

For some colors RGB, the corresponding colors $R_{p} G_{p} B_{p}$ obtained from Equation 6 are very close to the original RGB colors. We define the set $C_{\text {correct }}$ to be the set of colors RGB that are present in the image and for which the corresponding $R_{p} G_{p} B_{p}$ is within $1 \%$ of RGB. Note that $1 \%$ is a threshold which can be changed according to experiments. We define the set $C_{\text {incorrect }}$ to simply be the complement of $C_{\text {correct }}$ among all colors appearing in the image. In our daltonization method we want to achieve three goals:

1. Colors in $C_{\text {correct }}$ should not be changed.

2. Colors in $C_{\text {incorrect }}$ must be daltonized.

3. No color in $C_{\text {incorrect }}$ should be daltonized to a color that a protanope would perceive as similar to a color from $C_{\text {correct }}$.

Consequently, if the colors that we obtain from Equations 11 and 12 using matrix $M$ violate the third of the above requirements, we use an iterative algorithm, in which $M$ is repeatedly modified, until the third requirement is satisfied.

In order to specify the third requirement in a quantitative way, we define a predicate conflict $\left(R_{1} G_{1} B_{1}, R_{2} G_{2} B_{2}\right)$ as follows:

$\operatorname{conflict}\left(R_{1} G_{1} B_{1}, R_{2} G_{2} B_{2}\right)=\left\{\begin{array}{l}\text { true if }\left|R_{1}-R_{2}\right|<d,\left|G_{1}-G_{2}\right|<d,\left|B_{1}-B_{2}\right|<d, \\ \text { false otherwise. }\end{array}\right.$

where $d$ is an appropriately chosen threshold (in our experiments, $d=10$ ).

If $C_{1}$ and $C_{2}$ are sets of colors, we use notation setconflict $\left(C_{1}, C_{2}\right)$ for the predicate denoting whether there is a conflict between any color in $C_{1}$ and a color in $C_{2}$ :

$$
\operatorname{setconflict}\left(C_{1}, C_{2}\right)=\left\{\begin{array}{l}
\text { true if } \exists R_{1} G_{1} B_{1} \in C_{1}, R_{2} G_{2} B_{2} \in C_{2} \mid \operatorname{conflict}\left(R_{1} G_{1} B_{1}, R_{2} G_{2} B_{2}\right) \\
\text { false otherwise. }
\end{array}\right.
$$

Given a matrix $M$ and using Equations 11 and 12 we daltonize the colors of $C_{\text {incorrect }}$. We define $C_{\text {dalton }}$ to be the set of colors we obtain by daltonizing the colors of $C_{\text {incorrect }}$. Furthermore we define $C_{\text {protanope }}$ to be the set of colors we obtain by 
applying Equation 6 to the colors of $C_{\text {dalton }}$. Given the above definitions, our goal is to prevent any conflicts between colors in $C_{\text {protanope }}$ and $C_{\text {correct }}$.

However, if the image contains a large number of distinct colors, checking for conflicts can be too time consuming. Thus, we use clustering-based color quantization in order to reduce the number of colors we need to consider, thus obtaining significant speedups in the overall running time. Each color can be regarded as a point in a three dimensional space (e.g., RGB color space). Consequently, an image can be regarded as set (or a "cloud") of points in that space. Our goal is to create groups of points such that:

1. Points belonging to the same group must minimize a given distance function.

2. Points belonging to different groups must maximize a given distance function.

We achieve this clustering by using the Fuzzy-C-means algorithm $[13,5,2,10]$. An essential parameter for Fuzzy-C-means is parameter $C$, which is the number of clusters. In our problem, that is the number of colors with which we can describe more efficiently our image. The more colors we use the more accurate the image becomes but at the cost of increased running time. A good value for $C$ in our experiments was defined empirically $(C=100)$.

Next follows our pseudocode:

1. Read an image and run Fuzzy-C-means. We name cluster_centers the matrix containing our clusters' centers.

2. Classify each color from cluster_centers, as belonging to $C_{\text {correct }}$ or $C_{\text {incorrect }}$.

3. Apply color daltonization to every color in $C_{\text {incorrect }}$, as described in Equation 12 and name the resulting matrix $C_{\text {dalton }}$.

4. Run protanope simulation on every color in $C_{\text {dalton }}$, as described in Equation 6 and name the resulting matrix $C_{\text {protanope }}$.

5. If setconflict $\left(C_{\text {correct }}, C_{\text {protanope }}\right)$ is false, go to step 6 . Otherwise go back to step 2, after modifying Matrix M appropriately as described in Equation 16.

6. Produce the result image by replacing, in the original image, every color in $C_{\text {incorrect }}$ with the corresponding color in $C_{\text {dalton }}$.

The initial value $M_{0}$ given for matrix $M$ of Equation 12 (used in step 3) is defined as follows:

$$
M_{0}=\left[\begin{array}{ccc}
m_{1} & m_{2} & m_{3} \\
m_{4,0} & m_{5} & m_{6} \\
m_{7,0} & m_{8} & m_{9}
\end{array}\right]=\left[\begin{array}{ccc}
-1 & 0 & 0 \\
1 & 1 & 0 \\
1 & 0 & 1
\end{array}\right]
$$

When we execute step 3 for the first time we use matrix $M_{0}$. At the $t$-th iteration, matrix $M_{t}$ is obtained from $M_{t-1}$ as follows:

$$
M_{t+1}=\left[\begin{array}{ccc}
m_{1} & m_{2} & m_{3} \\
m_{4, t} & m_{5} & m_{6} \\
m_{7, t} & m_{8} & m_{9}
\end{array}\right]=\left[\begin{array}{ccc}
m_{1} & m_{2} & m_{3} \\
m_{4, t-1}-s & m_{5} & m_{6} \\
m_{7, t-1}+s & m_{8} & m_{9}
\end{array}\right]
$$

where $s$ is a predefined parameter ( $s=0.05$ in our experiments). 


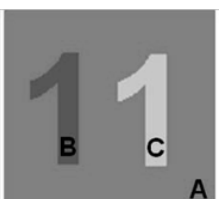

(a)

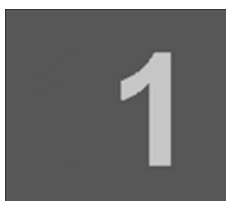

(b)

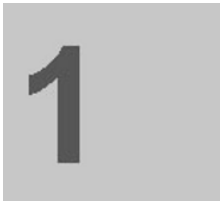

(c)

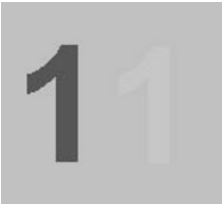

(d)

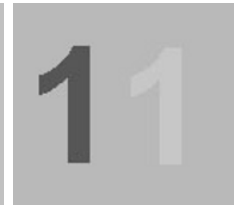

(e)

Fig. 2 (a) Original image, where $\mathrm{A}(255,51,204), \mathrm{B}(73,73,203)$ and $\mathrm{C}(193,193,255)$, (b) protanope perception of (a). Note that left " 1 " is not visible, (c) First iteration of our algorithm: note that now a protanope can't perceive right " 1 ", (d) Second iteration: note that the right "1" still isn't clear enough for a protanope, (e) Third iteration: note that now right "1" is visible to the protanope.

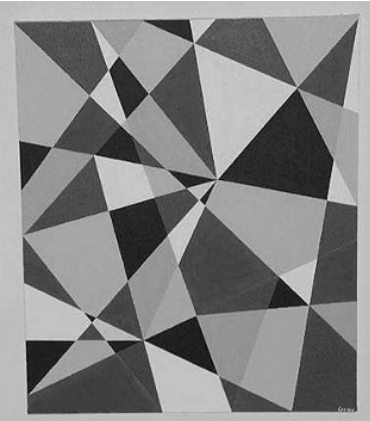

(a)

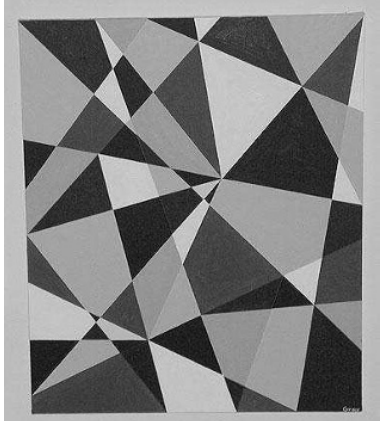

(b)

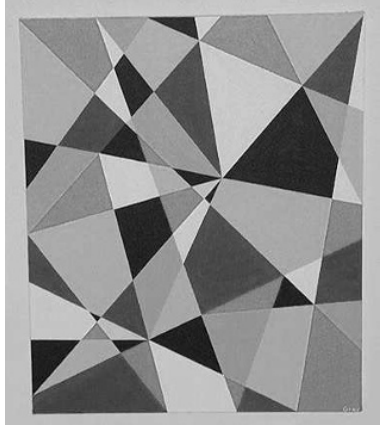

(c)

Fig. 3 (a) Original artwork "Prism", modern abstract painting in acrylics by Bruce Gray [6] , (b) Protanope perception of (a), (c) Protanope perception after running our algorithm.

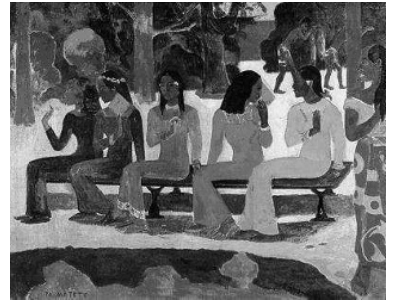

(a)

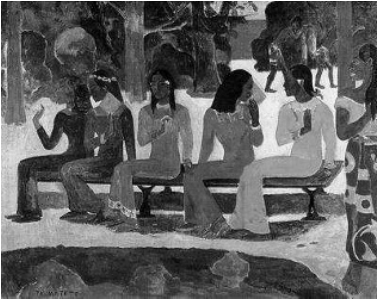

(b)

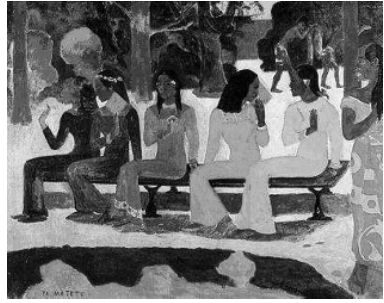

(c)

Fig. 4 Examples for Paul Gaugin's painting "Market Day" (a) Original image , (b) Protanope vision of (a) , (c) Protanope vision of (a) after running our algorithm

\section{Experiments}

First we test our algorithm on the image shown in Figure 2a. Note that in $2 b$, which shows how the image is perceived by a protanope, the left " 1 " is not visible. In 
Figure 2c, which shows the result after the first iteration, we are missing the right "1", whereas in Figure 2d, which shows the result of the second iteration, the right " 1 " is visible, but barely. Finally in Figure 2e, which shows the result (as perceived by a protanope) after the third iteration, both the left and the right " 1 " are visible.

In Figure 3a we show a modern abstract painting by Bruce Gray [6], called "Prism". Figure 3b shows the protanope's perception of the painting. We should note that red color is perceived as black, resulting in the protanope perceiving several pairs of adjacent red and black regions as single regions. In Figure $3 \mathrm{c}$ we show the result (as perceived by a protanope) after running our algorithm. We can see that the red and black regions that appeared merged in $3 \mathrm{~b}$ now have distinct colors.

Finally, in Figure 4a we show the painting "Market Day" by Paul Gaugin. Figure $4 \mathrm{~b}$ shows the protanope's perception of that painting. In Figure $4 \mathrm{c}$ we show the resulting image (as perceived by a protanope) after running our algorithm. We should note that several parts of the image structure are easier to perceive in Figure $4 \mathrm{c}$ compared to Figure 4b, including, e.g., the contrast in the bottom part of the image between the red and green colors that are shown in Figure 4a.

\section{Conclusions}

In this paper, a daltonization algorithm for people suffering from protanopia is proposed. More specifically, an intelligent iterative technique is described for the selection of the adaptation parameters. Computational time is drastically reduced due to the use of color quantization. One of our method's main advantages is that with minor modifications, it can be applied to other types of Color Vision Deficiency, such as deuteranopia, widely known as daltonism. An interesting future direction is extending the proposed method to handle video content in addition to static images.

\section{References}

1. C. Anagnostopoulos, I. Anagnostopoulos, G. Tsekouras, and C. Kalloniatis. Intelligent modification for the daltonization process of digitized paintings. In International Conference on Computer Vision Systems, 2007.

2. J.C. Bezdek and S.K. Pal. Fuzzy models for pattern recognition. methods that search for patterns in data. IEEE Press, 11:539, 1992.

3. Bob Dougherty and Alex Wade. Vischeck site. http://www.vischeck.com, last date of access $11 / 02 / 08$.

4. Onur Fidaner, Poliang Lin, and Nevran Ozguven. http://scien.stanford.edu/class/psych221/ projects/05/ofidaner/project_report.pdf, last date of access 11/02/08.

5. Klir G.J. Principles of uncertainty: What are they? why do we. need them? Fuzzy Sets and Systems, 74(1):15-31, 1995.

6. Bruce Gray. Bruce Gray site. http://www.brucegray.com, last date of access 11/02/08.

7. V.A. Kovalev. Towards image retrieval for eight percent of color-blind men. In International Conference on Pattern Recognition, volume 2, pages 943-946, 2004. 
8. Curtis E. Martin, J. O. Keller, Steven K. Rogers, and Matthew Kabrisky. Color blindness and a color human visual system model. IEEE Transactions on Systems, Man, and Cybernetics, Part A, 30(4):494-500, 2000.

9. Jeho Nam, Yong Man Ro, Youngsik Huh, and Munchurl Kim. Visual content adaptation according to user perception characteristics. IEEE Transactions On Multimedia, 7(3):435$445,2005$.

10. N. R. Pal and J. C. Bezdek. On clustering validity for the fuzzy c-means model. IEEE Transactions on Fuzzy Systems, 3:370-379, 1995.

11. F. Vinot, H. Brettel, and J. D. Mollon. Digital video colourmaps for checking the legibility of displays by dichromats. Color Research and Application, 24(4):243-252, 1999.

12. Seungji Yang and Yong Man Ro. Visual contents adaptation for color vision deficiency. In International Conference on Image Processing, volume 1, pages 453-456, 2003.

13. Lotfi A. Zadeh. Fuzzy sets. Information and Control, 8(3):338-353, 1965. 